\title{
Letter to the Editor on the article "Extraperitoneal vs. intraperitoneal route for permanent colostomy: a meta-analysis of 1071 patients"
}

\author{
L. F. Kroese ${ }^{1}$ (D) - J. Jeekel ${ }^{2}$ G. J. Kleinrensink ${ }^{2}$ · J. F. Lange ${ }^{1,3}$
}

Accepted: 13 October 2015 / Published online: 6 November 2015

(C) Springer-Verlag Berlin Heidelberg 2015

\section{Dear Editor:}

With great interest, we have read the meta-analysis published in the International Journal of Colorectal Disease, Volume 27, 59-64 (2012) by Lian et al. Great effort has been made to provide a complete overview of the effect of extraperitoneal colostomy formation on common surgical complications such as parastomal hernia, stoma prolapse, and bowel obstruction.

It is very interesting to see that the extraperitoneal route, although not often used, seems very promising, especially resulting in a lower parastomal hernia rate with an odds ratio of 0.41 ( $95 \%$ confidence interval 0.23 to $0.73, p=0.02$ ).
However, we would like to highlight that in our opinion, an incorrect forest plot (Fig. 4 in the original article) has been published. This figure is mentioned in the text, plotting the stoma prolapse rates of two studies, but in the forest plot, six studies are displayed. The event rates in the plot are identical to the rates in Fig. 2 displaying parastomal hernia rates.

With the forest plot being an excellent way of visualizing effect size and direction, it would be very interesting to see the correct figure to get a good picture of the results of the metaanalysis on the effect of extraperitoneal colostomy formation on stoma prolapse. Without the correct figure and event rates, one can only guess the specific study effect.

L. F. Kroese

lkroese@hotmail.com

1 Department of Surgery, Erasmus University Medical Center

Rotterdam, Room Ee-173, PO Box 2040, 3000, CA

Rotterdam, The Netherlands

2 Department of Neuroscience, Erasmus University Medical Center Rotterdam, Rotterdam, The Netherlands

3 Department of Surgery, Havenziekenhuis Rotterdam,

Rotterdam, The Netherlands 